\title{
25 Research Soure \\ Association of macular thickness with parapapillary atrophy in myopic eyes
}

\section{Helong Piao}

Chonnam National University Medical School and Hospital

Yue Guo

Chonnam National University Medical School and Hospital

Jun Young $\mathrm{Ha}$

Chonnam National University Medical School and Hospital

Mi Sun Sung

University College London Medical School

Sang Woo Park ( $D$ exo70@naver.com )

Chonnam National University Medical School and Hospital

\section{Research article}

Keywords: macular thickness, axial length, myopia, $\beta$-parapapillary atrophy with Bruch's membrane, $\beta$ parapapillary atrophy without Bruch's membrane

Posted Date: December 17th, 2019

DOl: https://doi.org/10.21203/rs.2.18777/v1

License: (c) (1) This work is licensed under a Creative Commons Attribution 4.0 International License.

Read Full License 


\section{Abstract}

\section{Background}

To investigate whether macular structure could be affected by axial elongation and to determine the association between macular intraretinal thickness and the microstructure of $\beta$-zone parapapillary atrophy (PPA) in myopic eyes.

\section{Methods}

The study recruited 113 healthy myopic subjects (113 eyes). Images of the macula, subfoveal choroid, and optic nerve head were acquired using spectral-domain optical coherence tomography (SD-OCT). An automatic segmentation algorithm was used to segment the macular images into 7 intraretinal layers. PPA widths with and without Bruch's membrane $\left(\mathrm{PPA}_{+\mathrm{BM}}\right.$ and $\mathrm{PPA}_{-\mathrm{BM}}$, respectively) were evaluated. Linear regression analysis was performed to evaluate the association between macular intraretinal thickness and axial length and the microstructure of PPA.

\section{Results}

An increase in axial length was associated with a decrease in whole macular thickness of the peripheral region and an increase in whole macular thickness of the central region. Thickness alterations of the macular intraretinal layers were most apparent in the peripheral region. A significant correlation was found between PPA ${ }_{-B M}$ width and macular intraretinal layer thickness, whereas no significant correlation was found between $\mathrm{PPA}_{+\mathrm{BM}}$ width and macular intraretinal layer thickness. Similar to axial length, as PPA. BM width increased, changes in macular intraretinal layer thickness were most pronounced in the peripheral region. Moreover, both $\mathrm{PPA}_{+\mathrm{BM}}$ and $\mathrm{PPA}_{-\mathrm{BM}}$ widths significantly correlated with subfoveal choroidal thickness.

\section{Conclusions}

Macular intraretinal layer thickness may be affected by PPA $_{-B M}$ width. These findings indicate that the microstructure of PPA should be considered when evaluating the macula in patient with myopia and glaucoma.

\section{Introduction}

Myopia is a very common ocular disorder worldwide. A recent meta-analysis predicted that myopia could be developed in nearly half of the world's population by 2050 [1]. In addition to the elongation of axial length, myopic eyes exhibit a series of pathophysiological changes, including thinning of the retina, choroid, and sclera [2-8]. With the progression of myopia, the potential risks of visual impairment become significantly higher and include retinal detachment, posterior staphyloma, cataract, choroidal neovascularization, and macular hole [9-10]. Several cross-sectional studies have suggested that myopic 
eyes were more susceptible to glaucoma than normal eyes [11, 12]. Therefore, understanding the mechanism of myopia and its underlying effect on the retina will be useful for the earlier diagnosis of myopia-related diseases.

On fundus photography, parapapillary atrophy (PPA) is classified into the peripheral a-zone and central $\beta$ zone. The $\alpha$-zone is an irregular pigmentation of the retinal pigment epithelium (RPE), while the $\beta$-zone is considered atrophy of the choroid and RPE. On the basis of the location of Bruch's membrane (BM) termination, $\beta$-zone PPA can be classified into $\beta$-parapapillary atrophy with and without BM $\left(\mathrm{PPA}_{+} \mathrm{BM}\right.$ and $\left.P P A_{-B M}\right)$. The region with $B M$ but without the RPE is $P P A_{+B M}$, and the region with neither the RPE nor BM is PPA - BM (or in other words Y-zone PPA) [13]. Several clinic-based studies have shown that $\mathrm{PPA}_{+} \mathrm{BM}$ was mostly associated with glaucoma, older age, and myopia. However, PPA BM $_{\text {B }}$ was associated with a longer axial length but not with glaucoma [14-17]. A previous study showed that optic nerve head (ONH) tilt was associated with $\mathrm{PPA}_{+\mathrm{BM}}$ and PPA $-\mathrm{BM}$ widths, which suggested that the microstructure of PPA might reflect the myopic remodeling of the $\mathrm{ONH}$ [18].

In macula, the evaluation of each intraretinal layer can give us the valuable information for the diagnosis and monitoring of several eye diseases including macular edema, glaucoma, and optic neuropathy and also the prediction of its variability is helpful for managing retinal diseases [19-21]. Of note, previous studies have reported that macular evaluation can be a valuable clinical parameter in managing patients with early to advanced glaucoma [22-25]. Jung et al. [22] reported that the evaluation of macular ganglion cell-inner plexiform layer (mGCIPL) thickness showed the similar diagnostic power to that of parapapillary retinal nerve fiber layer ( $p R N F L$ ) thicknesses in patient with normal tension glaucoma or primary open angle glaucoma. Sung et al. [25] showed that the assessment of macular thickness was useful parameter for detection of progression in patients with advanced glaucoma. Spectral-domain optical coherence tomography (SD-OCT) provides high-resolution images and enables the visualization of detailed morphological changes in each macular intraretinal layer and $\mathrm{ONH}$ [26].

Previous studies have investigated the association between age or several ocular parameters and the thickness of macular intraretinal layers [4, 27-29]. However, these studies yielded conflicting results. In this study, we hypothesized that the changes in the $\mathrm{ONH}$ might be associated with the alterations of the posterior pole. The purpose of this study was to determine whether macular intraretinal thickness was affected by axial elongation and to investigate the association between macular intraretinal thickness and the microstructure of PPA in myopic eyes.

\section{Methods}

\section{Subjects}

The study subjects were recruited from the Young Myopia Study of Chonnam National University Hospital, which is an ongoing cross-sectional study that commenced in January 2018. The present study was conducted according to the tenets of the Declaration of Helsinki and was approved by the 
Institutional Review Board of Chonnam National University Hospital. All patients provided written informed consent prior to enrollment in the study.

The Young Myopia Study enrolled consecutive participants who visited the general eye clinic for medical check-ups and met all the inclusion criteria and none of the exclusion criteria. Ocular examinations included measurements of best-corrected visual acuity (BCVA), intraocular pressure (IOP) using Goldmann applanation tonometry, autorefractometry, slit-lamp examination, and anterior chamber angle examination using gonioscopy. Central corneal thickness, keratometry, and axial length were measured using optical low-coherence reflectometry (Lenstar; Haag-Streit AG, Koeniz, Switzerland); ONH and pRNFL examinations were performed using color stereoscopic disc photography and red-free RNFL fundus photography; and visual field testing was performed using the Swedish Interactive Threshold Algorithm standard 30-2 perimetry with a Humphrey Field Analyzer (Carl Zeiss Meditec Inc., Dublin, CA, USA).

The inclusion criteria used in this study were as follows: ophthalmologically healthy subjects aged between 20 and 35 years old, spherical equivalent (SE) between -12.0 and -0.5 diopters, astigmatism within \pm 2 diopters, BCVA of $20 / 25$ or better, IOP $\leq 21 \mathrm{mmHg}$, normal ONHs on stereoscopic photographs (without a narrowed neuroretinal rim, abnormal tilt of the $\mathrm{ONH}$, parapapillary hemorrhage, or paleness), and the absence of any PRNFL abnormalities on red-free fundus photographs. Patients with visual field defects, a history of intraocular surgery, glaucoma, neurologic diseases, refractive surgery, other systemic diseases that affected ocular media opacities, or low-quality OCT images were excluded.

\section{Spectral-Domain Optical Coherence Tomography}

All subjects underwent traditional OCT imaging using SD-OCT (Heidelberg Spectralis SD-OCT; Spectralis software version 6.9.4; Heidelberg Engineering $\mathrm{GmbH}$, Heidelberg, Germany). One skilled operator performed all OCT scans. Ocular magnification effect was corrected using the formula provided by the manufacturer on the basis of the results of corneal radius, axial length, and focus setting during image acquisition. OCT images with insufficient quality (typically truncated B-scans and quality score $<30$ ) were excluded.

The macular cube volumetric scans with 49 raster lines, $20 \times 20^{\circ}$, interscan distance of $120 \mu \mathrm{m}$ between the 49 B-scans and centered on the fovea were acquired after mydriasis. The built-in software for automated segmentation of the intraretinal layers was applied, followed by manual inspection for obvious segmentation errors. The automatic segmentation algorithm divided the macular region into 9 sectors (Figure 1) and 7 layers (Figure 2), and they were named according to the guidelines of the Early Treatment Diabetic Retinopathy Study [30] and the International Nomenclature for Optical Coherence Tomography Panel [31]. For analysis, the macular region was divided into 3 concentric circles. The central region was a circle with a 1-mm diameter centered on the fovea. The pericentral region around the central region had a 3-mm diameter centered on the fovea. The peripheral region around the pericentral and central regions had a 6-mm diameter centered on the fovea. The 7 intraretinal layers included the RNFL, ganglion cell layer (GCL), inner plexiform layer (IPL), inner nuclear layer (INL), outer plexiform layer (OPL), 
outer nuclear layer (ONL), and RPE. The algorithm set the distance between the internal limiting membrane (ILM) and the middle of the RPE as the full retinal layer thickness.

\section{Optic Nerve Head Measurements}

The Heidelberg Spectralis OCT enhanced depth imaging (EDI) mode was used for the other ONH measurements. The $\mathrm{ONH}$ was scanned by centering a $15^{\circ} \times 10^{\circ}$ rectangular scan on the $\mathrm{ONH}$. Each OCT volume consisted of 49 serial horizontal B-scans (4.5-mm-long lines; 50 images averaged) spaced at approximately 63- $\mu \mathrm{m}$ intervals. Infrared (IR) fundus images were acquired simultaneously by using a confocal scanning laser ophthalmoscope. Three sections that passed through the $\mathrm{ONH}$ in the horizontal scans and upper and lower scans were selected, and all the study parameters were measured in each of these frames by 2 independent examiners (M.S.S and H.P), in a masked fashion.

Temporal $\beta$-zone PPA margin, Bruch's membrane opening (BMO), and disc margin were determined using IR fundus images. Temporal $\beta$-zone PPA margin and $\gamma$-zone PPA margin were also evaluated using IR fundus images. According to the location of BM termination, PPA was further divided into PPA $+B M$ and

$\mathrm{PPA}_{-\mathrm{BM}} ; \mathrm{PPA}_{+\mathrm{BM}}$ width was defined as the distance from the beginning of the RPE to BMO, and PPA ${ }_{-B M}$ width was defined as the distance from the temporal disc margin to the beginning of $\mathrm{BM}$. The measurement was performed using a previously described method [32, 33].

\section{Subfoveal Choroidal Thickness Measurements}

Subfoveal choroidal thickness was measured using the EDI technique with a 12-mm-length scan running through the fovea. The vertical distance from the hyperscattering outer border of the RPE to the inner border of the sclera at the fovea was defined as subfoveal choroidal thickness. Images with a signal strength below 70 or cases wherein the RPE and chorioscleral interface were not clearly identified were excluded from the analysis. The average of data from 2 independent examiners (M.S.S and H.P) were used in this study. In cases of disagreement, an open adjudication with a senior grader (S.W.P) was performed.

\section{Statistical Analysis}

IBM SPSS Statistics for Windows, Version 25.0 (IBM Corp., Armonk, NY, USA) was used for statistical analysis. Multiple linear regression analysis (adjusted for age and sex or age, sex, and axial length) was applied to determine the associations of macular thickness of each intraretinal layer with axial length and $\mathrm{PPA}_{+\mathrm{BM}}$ and PPA $\mathrm{BM}_{-\mathrm{M}}$ widths. Coefficients with $95 \%$ confidence intervals were presented. $P$ values $<0.05$ were considered statistically significant.

\section{Results}

The demographic and ocular characteristics of 113 eyes of 113 patients ( 74 men and 39 women) are shown in Table 1. The mean age of the patients was $26.8 \pm 3.2$ years (range, 20-35 years). The mean 
axial length and SE were $26.0 \pm 1.5 \mathrm{~mm}$ (range, 22.8-29.8 mm) and $-5.0 \pm 2.9$ diopter (range, -11.25 to -0.5 diopter), respectively. The mean IOP was $13.6 \pm 1.9 \mathrm{~mm} \mathrm{Hg}$ (range, $9-19 \mathrm{~mm} \mathrm{Hg}$ ).

Table 2 shows the results of the multiple linear regression analysis of the correlation between whole macular thickness and each intraretinal layer thickness and axial length. In evaluation of whole macular thickness, the central region ( $P=0.003$, unstandardized regression coefficient $\mathrm{B}: 3.585)$ increased with axial elongation. However, the peripheral region $(P<0.001, \mathrm{~B}$ : -3.313$)$ decreased with axial elongation.

In the central region, OPL was positively correlated with axial length $(P<0.001, \mathrm{~B}: 1.474)$. In the peripheral region, all intraretinal layer thicknesses were negatively correlated with axial length $(P<0.001, \mathrm{~B}:-1.051 ; P$ $<0.001$, B: $-0.771 ; P<0.001$, B: $-0.489 ; P=0.022$, B: $-0.263 ; P=0.013$, B: -0.903 ; and $P<0.001$, B: -3.313 for GCL, IPL, INL, OPL, ONL, and whole macular thicknesses, respectively), except for RNFL thickness ( $P<$ 0.012, B: 0.533). In addition, subfoveal choroidal thickness ( $P<0.001$, B: -29.116) significantly decreased with axial elongation. Among the 3 macular regions, the peripheral region was the most affected by axial elongation.

Table 3 shows the relationships between whole macular thickness and each intraretinal layer thickness and the microstructure of PPA, which were analyzed using multiple linear regression analysis. No significant correlation was found between $\mathrm{PPA}_{+\mathrm{BM}}$ width and whole macular thickness and each intraretinal layer thickness. In contrast, $\mathrm{PPA}_{-\mathrm{BM}}$ width had correlations with macular thicknesses. In the peripheral region, $\mathrm{GCL}, \mathrm{INL}, \mathrm{OPL}, \mathrm{ONL}$, and whole macular thicknesses were negatively correlated with PPA $_{-\mathrm{BM}}$ width $(P=0.010$, B: $-0.003 ; P=0.025$, B: $-0.002 ; P<0.001$, B: $-0.003 ; P=0.008, \mathrm{~B}:-0.007$; and $P<$ 0.001, B: -0.020 for GCL, INL, OPL, ONL, and whole macular thicknesses, respectively). In the pericentral region, INL and whole macular thicknesses were negatively correlated with $\mathrm{PPA}_{\mathrm{BM}}$ width $(P=0.023, \mathrm{~B}$ : -0.003; and $P=0.005$, B: -0.017 for INL and whole macular thickness, respectively). Subfoveal choroidal thickness was significantly correlated with both $\mathrm{PPA}_{+\mathrm{BM}}(P<0.001, \mathrm{~B}:-0.183)$ and PPA-${ }_{\mathrm{BM}}$ widths $(P<$ $0.001, B:-0.194)$. Figure 3 shows the association between intraretinal layer thickness (of the OPL and ONL in the peripheral region) and PPA $_{-B M}$ width. Similar to axial length, as PPA ${ }_{-B M}$ width increased, the regional changes in intraretinal layer thickness were more apparent in the peripheral region than in the other 2 regions.

\section{Discussion}

In this study, we evaluated the association of each macular layer thickness with axial length and the microstructure of PPA in myopic eyes. We found that central macular thickness positively correlated with axial length, whereas peripheral macular thickness and subfoveal choroidal thickness were negatively associated with axial length. Our study also revealed that PPA + BM width had no association with any macular layer thickness. However, PPA $_{-{ }_{-B M}}$ width had strong correlations with many macular layer thicknesses. Moreover, both PPA $+B M$ and PPA ${ }_{-B M}$ widths were negatively correlated with subfoveal 
choroidal thickness. To our knowledge, this is the first study demonstrating the association between macular thickness and the microstructure of PPA in myopic eyes.

In terms of regional changes, our study showed that macular thickness increased in the central region, but was stable in the pericentral region and decreased in the peripheral region, with a more obvious change in the longer eye. This finding is in accordance with those of previous studies [3,34-36]. The thickening of the central region might be due to the elongation of the eyeball, which was, in turn, caused by mechanical traction of the sclera. In such a condition, the extension of the sclera drives retinal thinning. Meanwhile, the tendency of the ILM to remain flat and the centripetal force of the posterior vitreous lead to the thickening of the central region [37].

In our study, the thickening of the central region was mainly attributed to the increase in OPL thickness, which was formed by dendrites [31]. Our result was consistent with that of a recent SD-OCT study on retinal thickness [38]. We speculate that the OPL comprises thousands of dendrites, and is more elastic than the ONL. As the axial length increases, the elevation of the central macular region would result in OPL deformation. However, some studies have reported different results about the correlations between macular thickness and axial length. The different study design and inclusion criteria might explain these conflicting results. For instance, in the study of Wakitani et al. [39] OCT scans were centered on the fovea with a scan length of $3 \mathrm{~mm}$, while the scan length was $6 \mathrm{~mm}$ in our study. High myopic eyes were excluded from the study by Ooto et al. [28], and hyperopic eyes were included in the study by Song et al. [40], while high myopia was included and hyperopia was excluded in our study. It is possible that the changes in retinal thickness in low-to-moderate myopia may be not enough to induce a morphological alteration.

In the peripheral region, the correlation between macular thickness and axial length was strong. All intraretinal layer thicknesses were correlated with axial length, and the thinning of the peripheral region was mainly because of the thinning of the GCL, IPL, and INL. This tendency was reported by Harb et al. [41], who stated that macular thinning in myopic eyes was more obvious in the peripheral region than in the other 2 regions.

In this study, RNFL thickness had a strong positive correlation with axial length in the pericentral and peripheral regions. Similar results had been described by Kim et al. [42] They proposed that with the expansion of the posterior pole, the retina could be pulled toward the temporal side, and in the RNFL, the compressed fiber bundles from the hemisphere on either side would be dragged to the horizontal raphe.

In terms of $\beta$-zone PPA, PPA + BM has been reported to be associated with older age or myopia [15-17]. But previous investigations have also revealed that $\mathrm{PPA}_{+\mathrm{BM}}$ was more significantly correlated with glaucoma $[13,14,16,43]$. Teng et al. [44] described that high IOP in patients with glaucoma may cause obstruction of the parapapillary choriocapillaris and, in turn, may lead to the degeneration of the RPE and adjacent cells. Similarly, Sullivan-Mee et al. [45] reported a significant correlation between juxtapapillary choroidal 
volume and PPA $+\mathrm{BM}$. The loss of RPE in the PPA $+\mathrm{BM}$ could be caused by disturbance of blood supply due to a thinned choroid, suggesting that $\mathrm{PPA}_{+\mathrm{BM}}$ may be associated with vascular compromise.

Conversely, PPA- $_{\mathrm{BM}}$ has been known to be associated with mainly axial elongation. Recent studies showed that an increase in axial length could lead to remodeling of the parapapillary region, and the backward pull via the optic nerve may act on the posterior sclera, leading to the formation and development of PPA ${ }_{-B M}[15,46,47]$. Lee et al. [48] reported that the development of PPA-BM reflected scleral overgrowth when compared with the inner retinal structures in the growing eye. Hence, $\mathrm{PPA}_{-\mathrm{BM}}$ is strongly correlated with axial length, and this might account for the association between PPA ${ }_{-B M}$ and myopia. Chui et al. [49] proved that retinal extension may not mirror scleral growth when measuring 2 parameters of the posterior pole, and the retina could slide to the temporal side during eye growth. Collectively, we suggest that $\mathrm{PPA}_{+\mathrm{BM}}$ is primarily involved in localized impairment of parapapillary choriocapillaris circulation and that PPA ${ }_{-B M}$ mainly reflects the broad posterior pole change caused by optic nerve traction due to axial elongation.

Multiple linear regression analysis revealed that $\mathrm{PPA}_{+\mathrm{BM}}$ width had no association with macular layer thickness. However, the relationship between PPA $_{-B M}$ width and macular layer thickness was obvious. In addition, PPA $_{-B_{M}}$ width had a stronger correlation with several macular intraretinal layer thicknesses than with axial length. This result led us to presume that as a monitoring marker, PPA ${ }_{-B M}$ might reflect changes of macular microstructure.

The choroids can be thinner as the axial length increases [5, 6]. In our study, subfoveal choroidal thickness was significantly correlated with both $\mathrm{PPA}_{+\mathrm{BM}}$ and $\mathrm{PPA}_{-\mathrm{BM}}$ widths. Interestingly, $\mathrm{PPA}^{-{ }_{\mathrm{BM}}}$ width was correlated with both subfoveal choroidal thickness and macular thickness. Nevertheless, PPA $+B M$ width was correlated with subfoveal choroidal thickness, but not with macular thickness. The development of PPA- ${ }_{\mathrm{BM}}$ reflects that axial elongation and broad posterior pole changes may be involved in the sclera, choroid, and retina. Hence, PPA- $^{-}{ }_{\mathrm{BM}}$ is associated with both subfoveal choroidal thickness and macular thickness. As mentioned above, $\mathrm{PPA}_{+\mathrm{BM}}$ could be associated with disturbance of blood supply due to a thinned choroid, not with retina. That might explain why PPA $+B M$ was correlated with choroid, but not with macula.

Our study has several limitations. First, we could not account for optic disc tilt, which may be an important measurement indicator of the $\mathrm{ONH}$. Previous studies have shown that optic disc tilt is correlated with the perfusion of the foveal zone [50] and RNFL thickness [51]. A more important association between optic disc tilt and macular thickness may exist. Second, observing the changes of the equatorial retina in detail was difficult, but knowledge about this could let us further understand the progression trend of myopia. Finally, we did not measure the lamina cribrosa and sclera. For understanding the underlying mechanism of myopia, future studies should assess the lamina cribrosa and sclera in myopic eyes. 
In conclusion, our study showed that macular intraretinal layer thicknesses have significant associations with axial length and PPA-${ }_{\text {BM }}$ width. With an increase in axial length, whole macular thickness in the peripheral region decreased, while whole macular thickness in the central region increased. PPA $^{-}{ }_{\text {BM }}$ width was correlated with several macular intraretinal layer thicknesses; however, no correlation was observed between $\mathrm{PPA}_{+\mathrm{BM}}$ width and macular intraretinal layer thickness. Given the clinical significance of macular evaluation in managing glaucoma, the microstructure of PPA, especially $\mathrm{PPA}^{-}{ }_{\mathrm{BM}}$, should be considered when evaluating the macula in patient with myopia and glaucoma.

\section{List Of Abbreviations}

PPA: Parapapillary atrophy; SD-OCT: Spectral-domain optical coherence tomography; BM: Bruch's membrane; PPA+BM: $\beta$-parapapillary atrophy with Bruch's membrane; PPA-BM: $\beta$-parapapillary atrophy without Bruch's membrane; ONH: Optic nerve head; mGCIPL: Macular ganglion cell-inner plexiform layer; pRNFL: Parapapillary retinal nerve fiber layer; BCVA: Best corrected visual acuity; IOP: Intraocular pressure; SE: Spherical equivalent; RNFL: Retinal nerve fiber layer; GCL: Ganglion cell layer; IPL: Inner plexiform layer; INL: Inner nuclear layer; OPL: Outer plexiform layer; ONL: Outer nuclear layer; RPE: Retinal pigment epithelium; ILM: Internal limiting membrane; EDI: Enhanced depth imaging; IR: Infrared; BMO: Bruch's membrane opening.

\section{Declarations}

\section{Funding}

No funding was received by any of the authors in the writing of this manuscript.

\section{Availability of data and materials}

The datasets analyzed in this study are available from the corresponding author (Sang Woo Park, exo70@naver.com) upon reasonable request.

\section{Authors' contributions}

HP, MSS and SWP designed the study. HP, YG and JYH collected the data and involved in the analysis. HP wrote the first draft of the manuscript. HP, MSS and SWP reviewed and revised the manuscript and produced the final version. All authors have read and approved the final manuscript.

\section{Ethics approval and consent to participate}

Ethics committee approval was obtained from the Institutional Review Board of Chonnam National University Medical School and Hospital. We carried out the study according to the tenets of the Declaration of Helsinki involving human participants and the approved guidelines.

\section{Consent for publication}


Not applicable.

\section{Competing interests}

The authors declare that they have no competing interests.

\section{Acknowledgements}

Not applicable.

\section{References}

1. Holden BA, Fricke TR, Wilson DA, et al. Global Prevalence of Myopia and High Myopia and Temporal Trends from 2000 through 2050. Ophthalmology. 2016;123:1036-42.

2. Chen S, Wang B, Dong N, Ren X, Zhang T, Xiao L. Macular measurements using spectral-domain optical coherence tomography in Chinese myopic children. Invest Ophthalmol Vis Sci. 2014;55:74106.

3. Lam DS, Leung KS, Mohamed S, et al. Regional variations in the relationship between macular thickness measurements and myopia. Invest Ophthalmol Vis Sci. 2007;48:376-82.

4. Szigeti A, Tátrai E, Varga BE, et al. The Effect of Axial Length on the Thickness of Intraretinal Layers of the Macula. PLoS One. 2015;10:e0142383.

5. Harb E, Hyman L, Gwiazda J, et al. Choroidal Thickness Profiles in Myopic Eyes of Young Adults in the Correction of Myopia Evaluation Trial Cohort. Am J Ophthalmol. 2015;160:62-71.e2.

6. Flores-Moreno I, Lugo F, Duker JS, Ruiz-Moreno JM. The relationship between axial length and choroidal thickness in eyes with high myopia. Am J Ophthalmol. 2013;155:314-9.e1.

7. Wong CW, Phua V, Lee SY, Wong TY, Cheung CM. Is Choroidal or Scleral Thickness Related to Myopic Macular Degeneration?. Invest Ophthalmol Vis Sci. 2017;58:907-13.

8. Vurgese S, Panda-Jonas S, Jonas JB. Scleral thickness in human eyes. PLoS One. 2012;7:e29692.

9. Saw SM, Gazzard G, Shih-Yen EC, Chua WH. Myopia and associated pathological complications. Ophthalmic Physiol Opt. 2005;25:381-91.

10. Saw SM. How blinding is pathological myopia?. Br J Ophthalmol. 2006;90:525-6.

11. Czudowska MA, Ramdas WD, Wolfs RC, et al. Incidence of glaucomatous visual field loss: a ten-year follow-up from the Rotterdam Study. Ophthalmology. 2010;117:1705-12.

12. Suzuki $Y$, Iwase A, Araie $M$, et al. Risk factors for open-angle glaucoma in a Japanese population: the Tajimi Study. Ophthalmology. 2006;113:1613-7.

13. Lee SH, Lee EJ, Kim TW. Topographic Correlation Between Juxtapapillary Choroidal Thickness and Microstructure of Parapapillary Atrophy. Ophthalmology. 2016;123:1965-73.

14. Jonas JB, Jonas SB, Jonas RA, et al. Parapapillary atrophy: histological gamma zone and delta zone. PLoS One. 2012;7:e47237. 
15. Zhang Q, Wang YX, Wei WB, Xu L, Jonas JB. Parapapillary Beta Zone and Gamma Zone in a Healthy Population: The Beijing Eye Study 2011. Invest Ophthalmol Vis Sci. 2018;59:3320-9.

16. Miki A, Ikuno Y, Weinreb RN, et al. Measurements of the parapapillary atrophy zones in en face optical coherence tomography images. PLoS One. 2017;12:e0175347.

17. Kim M, Kim TW, Weinreb RN, Lee EJ. Differentiation of parapapillary atrophy using spectral-domain optical coherence tomography. Ophthalmology. 2013;120:1790-7.

18. Marsh-Tootle WL, Harb E, Hou W, et al. Optic Nerve Tilt, Crescent, Ovality, and Torsion in a MultiEthnic Cohort of Young Adults With and Without Myopia. Invest Ophthalmol Vis Sci. 2017;58:315871.

19. Barham R, El Rami H, Sun JK, Silva PS. Evidence-Based Treatment of Diabetic Macular Edema. Semin Ophthalmol. 2017;32:56-66.

20. Hood DC. Improving our understanding, and detection, of glaucomatous damage: an approach based upon optical coherence tomography (OCT). Prog Retin Eye Res. 2017;57:46-75.

21. Huang S, Chen Q, Ma Q, Liu X, Lu F, Shen M. Three-dimensional characteristics of four macular intraretinal layer thicknesses in symptomatic and asymptomatic carriers of G11778A mutation with leber's hereditary optic neuropathy. Retina. 2016;36:2409-18.

22. Jung HH, Sung MS, Heo H, Park SW. Macular inner plexiform and retinal nerve fiber layer thickness in glaucoma. Optom Vis Sci. 2014;91:1320-7.

23. Sung MS, Heo H, Park SW. Structure-function Relationship in Advanced Glaucoma After Reaching the RNFL Floor. J Glaucoma. 2019;28:1006-11.

24. Park JW, Jung HH, Heo H, Park SW. Validity of the temporal-to-nasal macular ganglion cell-inner plexiform layer thickness ratio as a diagnostic parameter in early glaucoma. Acta Ophthalmol. 2015;93:e356-65.

25. Sung KR, Sun JH, Na JH, Lee JY, Lee Y. Progression detection capability of macular thickness in advanced glaucomatous eyes. Ophthalmology. 2012;119:308-13.

26. Terry L, Cassels N, Lu K, et al. Automated Retinal Layer Segmentation Using Spectral Domain Optical Coherence Tomography: Evaluation of Inter-Session Repeatability and Agreement between Devices. PLoS One. 2016;11:e0162001.

27. Demirkaya N, van Dijk HW, van Schuppen SM, et al. Effect of age on individual retinal layer thickness in normal eyes as measured with spectral-domain optical coherence tomography. Invest Ophthalmol Vis Sci. 2013;54:4934-40.

28. Ooto S, Hangai M, Tomidokoro A, et al. Effects of age, sex, and axial length on the three-dimensional profile of normal macular layer structures. Invest Ophthalmol Vis Sci. 2011;52:8769-79.

29. Liu X, Shen M, Yuan Y, et al. Macular Thickness Profiles of Intraretinal Layers in Myopia Evaluated by Ultrahigh-Resolution Optical Coherence Tomography. Am J Ophthalmol. 2015;160:53-61.e2.

30. Early Treatment Diabetic Retinopathy Study design and baseline patient characteristics. ETDRS report number 7. Ophthalmology. 1991;98:741-56. 
31. Staurenghi G, Sadda S, Chakravarthy U, Spaide RF, International Nomenclature for Optical Coherence Tomography (IN•OCT) Panel.. Proposed lexicon for anatomic landmarks in normal posterior segment spectral-domain optical coherence tomography: the IN•OCT consensus. Ophthalmology. 2014;121:1572-8.

32. Yamada H, Akagi T, Nakanishi $\mathrm{H}$, et al. Microstructure of Peripapillary Atrophy and Subsequent Visual Field Progression in Treated Primary Open-Angle Glaucoma. Ophthalmology. 2016;123:542-51.

33. Kim YW, Lee EJ, Kim TW, Kim M, Kim H. Microstructure of $\beta$-zone parapapillary atrophy and rate of retinal nerve fiber layer thinning in primary open-angle glaucoma. Ophthalmology. 2014;121:1341-9.

34. Hwang YH, Kim YY. Macular thickness and volume of myopic eyes measured using spectral-domain optical coherence tomography. Clin Exp Optom. 2012;95:492-8.

35. Choovuthayakorn J, Laowong T, Watanachai N, Patikulsila D, Chaikitmongkol V. Spectral-domain optical coherence tomography of macula in myopia. Int Ophthalmol. 2016;36:319-25.

36. Zhao MH, Wu Q, Hu P, Jia LL. Macular Thickness in Myopia: An OCT Study of Young Chinese Patients. Curr Eye Res. 2016;41:1373-8.

37. Wu PC, Chen YJ, Chen $\mathrm{CH}$, et al. Assessment of macular retinal thickness and volume in normal eyes and highly myopic eyes with third-generation optical coherence tomography. Eye (Lond). 2008;22:551-5.

38. Invernizzi A, Pellegrini M, Acquistapace A, et al. Normative Data for Retinal-Layer Thickness Maps Generated by Spectral-Domain OCT in a White Population. Ophthalmol Retina. 2018;2:808-815.e1.

39. Wakitani Y, Sasoh M, Sugimoto M, Ito Y, Ido M, Uji Y. Macular thickness measurements in healthy subjects with different axial lengths using optical coherence tomography. Retina. 2003;23:177-82.

40. Song WK, Lee SC, Lee ES, Kim CY, Kim SS. Macular thickness variations with sex, age, and axial length in healthy subjects: a spectral domain-optical coherence tomography study. Invest Ophthalmol Vis Sci. 2010;51:3913-8.

41. Harb E, Hyman L, Fazzari M, Gwiazda J, Marsh-Tootle W, COMET Study Group.. Factors associated with macular thickness in the COMET myopic cohort. Optom Vis Sci. 2012;89:620-31.

42. Kim MJ, Lee EJ, Kim TW. Peripapillary retinal nerve fibre layer thickness profile in subjects with myopia measured using the Stratus optical coherence tomography. Br J Ophthalmol. 2010;94:11520.

43. Vianna JR, Malik R, Danthurebandara VM, et al. Beta and Gamma Peripapillary Atrophy in Myopic Eyes With and Without Glaucoma. Invest Ophthalmol Vis Sci. 2016;57:3103-11.

44. Teng CC, De Moraes CG, Prata TS, et al. The region of largest $\beta$-zone parapapillary atrophy area predicts the location of most rapid visual field progression. Ophthalmology. 2011;118:2409-13.

45. Sullivan-Mee M, Patel NB, Pensyl D, Qualls C. Relationship Between Juxtapapillary Choroidal Volume and Beta-Zone Parapapillary Atrophy in Eyes With and Without Primary Open-Angle Glaucoma. Am J Ophthalmol. 2015;160:637-47.e1. 
46. Chang MY, Shin A, Park J, et al. Deformation of Optic Nerve Head and Peripapillary Tissues by Horizontal Duction. Am J Ophthalmol. 2017;174:85-94.

47. Jonas JB, Fang Y, Weber P, Ohno-Matsui K. PARAPAPILLARY GAMMA AND DELTA ZONES IN HIGH MYOPIA. Retina. 2018;38:931-8.

48. Lee KM, Choung HK, Kim M, Oh S, Kim SH. Change of $\beta$-Zone Parapapillary Atrophy During Axial Elongation: Boramae Myopia Cohort Study Report 3. Invest Ophthalmol Vis Sci. 2018;59:4020-30.

49. Chui TY, Zhong Z, Burns SA. The relationship between peripapillary crescent and axial length: Implications for differential eye growth. Vision Res. 2011;51:2132-8.

50. Sung MS, Lee TH, Heo H, Park SW. Association Between Optic Nerve Head Deformation and Retinal Microvasculature in High Myopia. Am J Ophthalmol. 2018;188:81-90.

51. Hwang YH, Yoo C, Kim YY. Myopic optic disc tilt and the characteristics of peripapillary retinal nerve fiber layer thickness measured by spectral-domain optical coherence tomography. J Glaucoma. 2012;21:260-5.

\section{Tables}

TABLE 1. Demographics and ocular parameters of study subjects

\begin{tabular}{lccc}
\hline & \multicolumn{3}{c}{ Number of subjuects $(\mathrm{n}=113)$} \\
\cline { 2 - 4 } & Mean & SD & Range \\
\hline Age, y & 26.8 & 3.2 & $20-35$ \\
Sex, male/female & $74 / 39$ & & \\
Axial length, mm & 26.0 & 1.5 & $22.8-29.8$ \\
IOP, mm Hg & 13.6 & 1.9 & $9-19$ \\
Spherical equivalent, diopter & -5.0 & 2.9 & $-11.25--0.5$ \\
Average keratometry, diopter & 42.8 & 1.1 & $39.9-45.7$ \\
Central corneal thickness, $\mu \mathrm{m}$ & 554.3 & 36.2 & $484-674$ \\
PPA $_{+ \text {BM } \text { width, } \mu \mathrm{m}}$ & 180.5 & 174.8 & $0-868$ \\
PPA-BM width, $\mu \mathrm{m}$ & 284.5 & 216.3 & $0-706$ \\
Subfoveal choroidal thickness, $\mu \mathrm{m}$ & 268.0 & 85.4 & $80-416$ \\
Average pRNFL thickness, $\mu \mathrm{m}$ & 89.7 & 8.3 & $72-109$ \\
\hline
\end{tabular}

$\mathrm{IOP}=$ intraocular pressure; $\mathrm{PPA}_{+\mathrm{BM}}=\beta$-parapapillary atrophy with Bruch's membrane; $\mathrm{PPA}_{-\mathrm{BM}}=\beta$-parapapillary atrophy without Bruch's membrane; $\mathrm{pRNFL}=$ parapapillary retinal nerve fiber layer.

TABLE 2. Associations of the thickness of whole macula and each macular layer with axial length in myopic eyes. 


\begin{tabular}{|c|c|c|}
\hline & \multicolumn{2}{|l|}{ Axial Length } \\
\hline & B $(95 \%$ CI $)$ & $P$ Value ${ }^{\mathrm{a}}$ \\
\hline \multicolumn{3}{|l|}{ Central region } \\
\hline OPL & $1.474(0.726-2.222)$ & $<0.001$ \\
\hline ONL & $-0.983(-2.381-0.415)$ & 0.166 \\
\hline Whole & $3.585(1.215-5.955)$ & 0.003 \\
\hline \multicolumn{3}{|l|}{ Pericentral region } \\
\hline NFL & $0.540(0.339-0.740)$ & $<0.001$ \\
\hline GCL & $0.056(-0.342-0.455)$ & 0.779 \\
\hline IPL & $-0.023(-0.258-0.212)$ & 0.846 \\
\hline INL & $-0.156(-0.508-0.196)$ & 0.382 \\
\hline OPL & $0.341(-0.271-0.952)$ & 0.272 \\
\hline ONL & $-1.127(-2.240--0.014)$ & 0.047 \\
\hline Whole & $-0.952(-2.712-0.807)$ & 0.286 \\
\hline \multicolumn{3}{|l|}{ Peripheral region } \\
\hline NFL & $0.533(0.118-0.948)$ & 0.012 \\
\hline GCL & $-1.051(-1.362--0.740)$ & $<0.001$ \\
\hline IPL & $-0.771(-1.013--0.528)$ & $<0.001$ \\
\hline INL & $-0.489(-0.727--0.250)$ & $<0.001$ \\
\hline OPL & $-0.263(-0.487--0.038)$ & 0.022 \\
\hline ONL & $-0.903(-1.615--0.192)$ & 0.013 \\
\hline Whole & $-3.313(-4.849--1.777)$ & $<0.001$ \\
\hline Subfoveal choroid & $-29.116(-38.207--20.024)$ & $<0.001$ \\
\hline
\end{tabular}

$\mathrm{B}=$ Unstandardized coefficient $\mathrm{B} ; \mathrm{NFL}=$ nerve fiber layer; GCL = ganglion cell layer, $\mathrm{IPL}=$ inner plexiform layer; INL = inner nuclear layer; OPL = outer plexiform layer; ONL = outer nuclear layer.

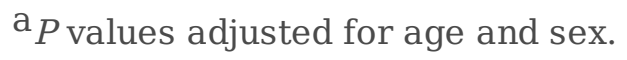

Values with statistical significance are shown in bold.

TABLE 3. Associations of the thickness of whole macula and each macular layer with microstructure of PPA. 


\begin{tabular}{|c|c|c|c|c|c|c|}
\hline & \multicolumn{3}{|c|}{$\overline{\mathrm{PPA}+\mathrm{BM}}$} & \multicolumn{3}{|c|}{$\overline{\mathrm{PPA}_{\mathrm{BM}}}$} \\
\hline & $\bar{B}(95 \%$ CI $)$ & $P$ value $^{\mathrm{a}}$ & $P$ value ${ }^{\mathrm{b}}$ & B (95\% CI) & $P$ value $^{\mathrm{a}}$ & $P$ value $^{\mathrm{b}}$ \\
\hline \multicolumn{7}{|l|}{ Central region } \\
\hline OPL & $\begin{array}{c}0.004(-0.003- \\
0.011)\end{array}$ & 0.210 & 0.980 & $\begin{array}{c}0.010(-0.005- \\
0.016)\end{array}$ & $<0.001$ & 0.018 \\
\hline ONL & $\begin{array}{c}-0.005(-0.018- \\
0.007)\end{array}$ & 0.387 & 0.680 & $\begin{array}{c}-0.016(-0.026- \\
-0.007)\end{array}$ & 0.001 & 0.003 \\
\hline Whole & $\begin{array}{c}0.006(-0.016- \\
0.027)\end{array}$ & 0.599 & 0.600 & $\begin{array}{c}0.004(-0.014- \\
0.021)\end{array}$ & 0.685 & 0.266 \\
\hline \multicolumn{7}{|l|}{ Pericentral region } \\
\hline NFL & $\begin{array}{c}0.001(-0.001- \\
0.003)\end{array}$ & 0.378 & 0.526 & $\begin{array}{c}0.001(-0.001- \\
0.002)\end{array}$ & 0.308 & 0.187 \\
\hline GCL & $\begin{array}{c}-0.002(-0.006- \\
0.001)\end{array}$ & 0.211 & 0.154 & $\begin{array}{c}-0.002(-0.005- \\
0.001)\end{array}$ & 0.161 & 0.081 \\
\hline IPL & $\begin{array}{c}-0.002(-0.004- \\
0.000)\end{array}$ & 0.097 & 0.100 & $\begin{array}{c}-0.001(-0.002- \\
0.001)\end{array}$ & 0.269 & 0.277 \\
\hline INL & $\begin{array}{c}-0.003(-0.006- \\
0.000)\end{array}$ & 0.094 & 0.140 & $\begin{array}{c}-0.003(-0.005- \\
0.000)\end{array}$ & 0.023 & 0.040 \\
\hline OPL & $\begin{array}{c}0.000(-0.005- \\
0.006)\end{array}$ & 0.931 & 0.824 & $\begin{array}{c}0.000(-0.004- \\
0.004)\end{array}$ & 0.886 & 0.303 \\
\hline ONL & $\begin{array}{c}0.001(-0.008- \\
0.011)\end{array}$ & 0.772 & 0.342 & $\begin{array}{c}-0.009(-0.016- \\
-0.001)\end{array}$ & 0.025 & 0.129 \\
\hline Whole & $\begin{array}{c}-0.003(-0.018- \\
0.012)\end{array}$ & 0.673 & 0.935 & $\begin{array}{c}-0.017(-0.028- \\
-0.005)\end{array}$ & 0.005 & 0.011 \\
\hline \multicolumn{7}{|l|}{ Peripheral region } \\
\hline NFL & $\begin{array}{c}0.004(0.001- \\
0.008)\end{array}$ & 0.056 & 0.088 & $\begin{array}{c}0.000(-0.003- \\
0.003)\end{array}$ & 0.846 & 0.294 \\
\hline GCL & $\begin{array}{c}-0.001(-0.004- \\
0.002)\end{array}$ & 0.421 & 0.274 & $\begin{array}{c}-0.003(-0.006- \\
-0.001)\end{array}$ & 0.010 & 0.931 \\
\hline IPL & $\begin{array}{c}-0.001(-0.003- \\
0.002)\end{array}$ & 0.649 & 0.156 & $\begin{array}{c}-0.002(-0.004- \\
0.000)\end{array}$ & 0.074 & 0.447 \\
\hline INL & $\begin{array}{c}-0.001(-0.003- \\
0.001)\end{array}$ & 0.407 & 0.719 & $\begin{array}{c}-0.002(-0.004- \\
0.000)\end{array}$ & 0.025 & 0.519 \\
\hline OPL & $\begin{array}{c}-0.002(-0.004- \\
0.000)\end{array}$ & 0.100 & 0.333 & $\begin{array}{c}-0.003(-0.004- \\
-0.002)\end{array}$ & $<0.001$ & 0.002 \\
\hline ONL & $\begin{array}{c}0.000(-0.006- \\
0.007)\end{array}$ & 0.890 & 0.338 & $\begin{array}{c}-0.007(-0.011- \\
-0.002)\end{array}$ & 0.008 & 0.015 \\
\hline Whole & $\begin{array}{c}-0.001(-0.016- \\
0.013)\end{array}$ & 0.859 & 0.245 & $\begin{array}{c}-0.020(-0.031- \\
-0.009)\end{array}$ & $<0.001$ & 0.040 \\
\hline Subfoveal choroid & $\begin{array}{c}-0.183(-0.267- \\
-0.098)\end{array}$ & $<0.001$ & 0.004 & $\begin{array}{c}-0.194(-0.257- \\
-0.132)\end{array}$ & $<0.001$ & $<0.001$ \\
\hline
\end{tabular}

$\mathrm{PPA}=$ parapapillary atrophy; $\mathrm{PPA}_{+\mathrm{BM}}=\beta$-parapapillary atrophy with Bruch's membrane; $\mathrm{PPA}_{-\mathrm{BM}}=\beta$ parapapillary atrophy without Bruch's membrane; $\mathrm{B}=$ Unstandardized coefficient; NFL = nerve fiber layer; GCL = ganglion cell layer; IPL = inner plexiform layer; INL = inner nuclear layer; OPL = outer plexiform layer; $\mathrm{ONL}=$ outer nuclear layer.

a $P$ value adjusted for age and sex.

$\mathrm{b}_{P}$ value adjusted for age, sex, and axial length.

Values with statistical significance are shown in bold. 


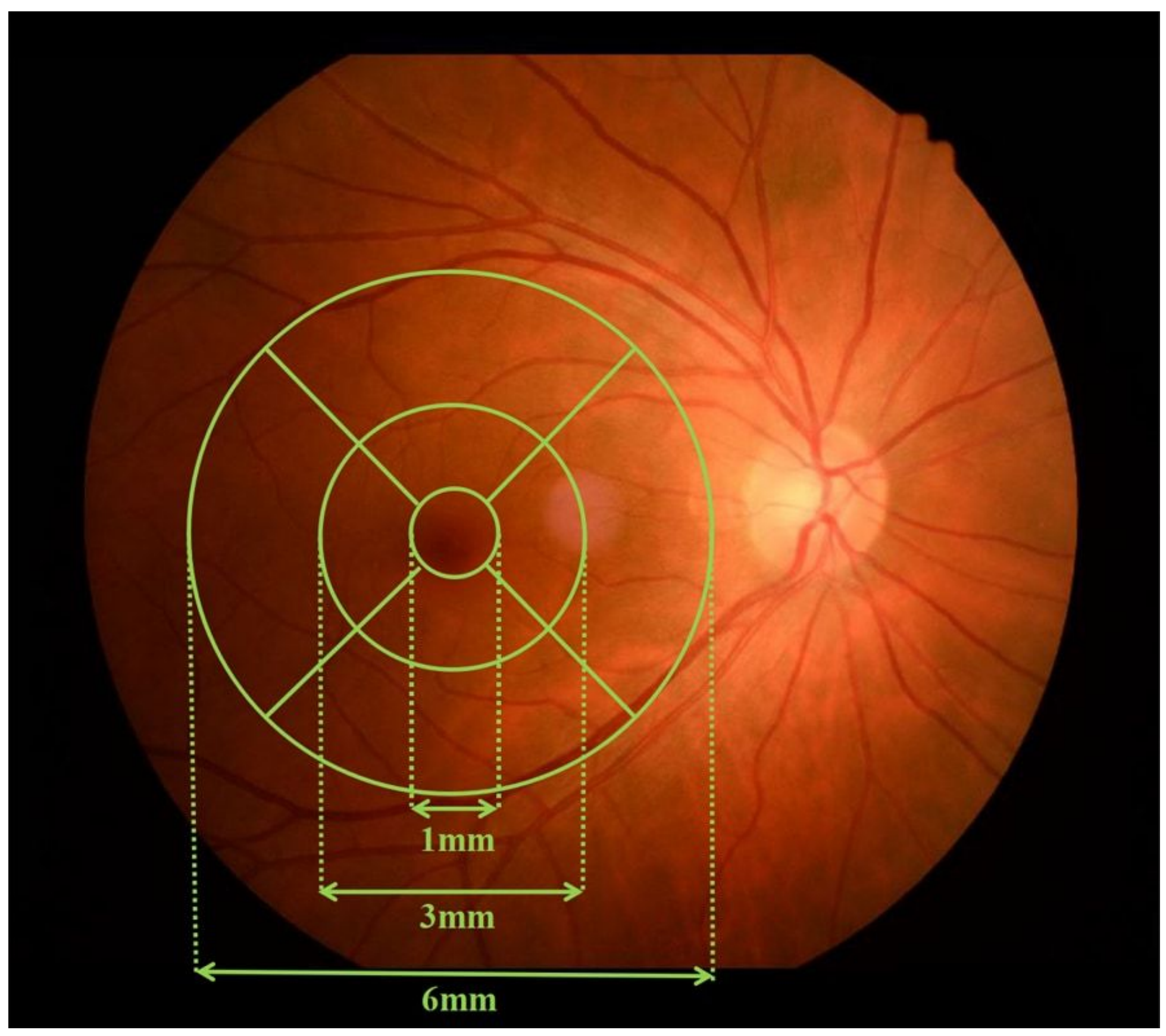

\section{Figure 1}

Analysis of the macular region by using Heidelberg Spectralis spectral-domain optical coherence tomography. The macula is subdivided into 3 concentric circles. The central region is a circle with a 1-mm diameter centered on the fovea. The pericentral region around the central region has a 3-mm diameter centered on the fovea. The peripheral region around the pericentral and central regions has a $6-\mathrm{mm}$ diameter centered on the fovea. 


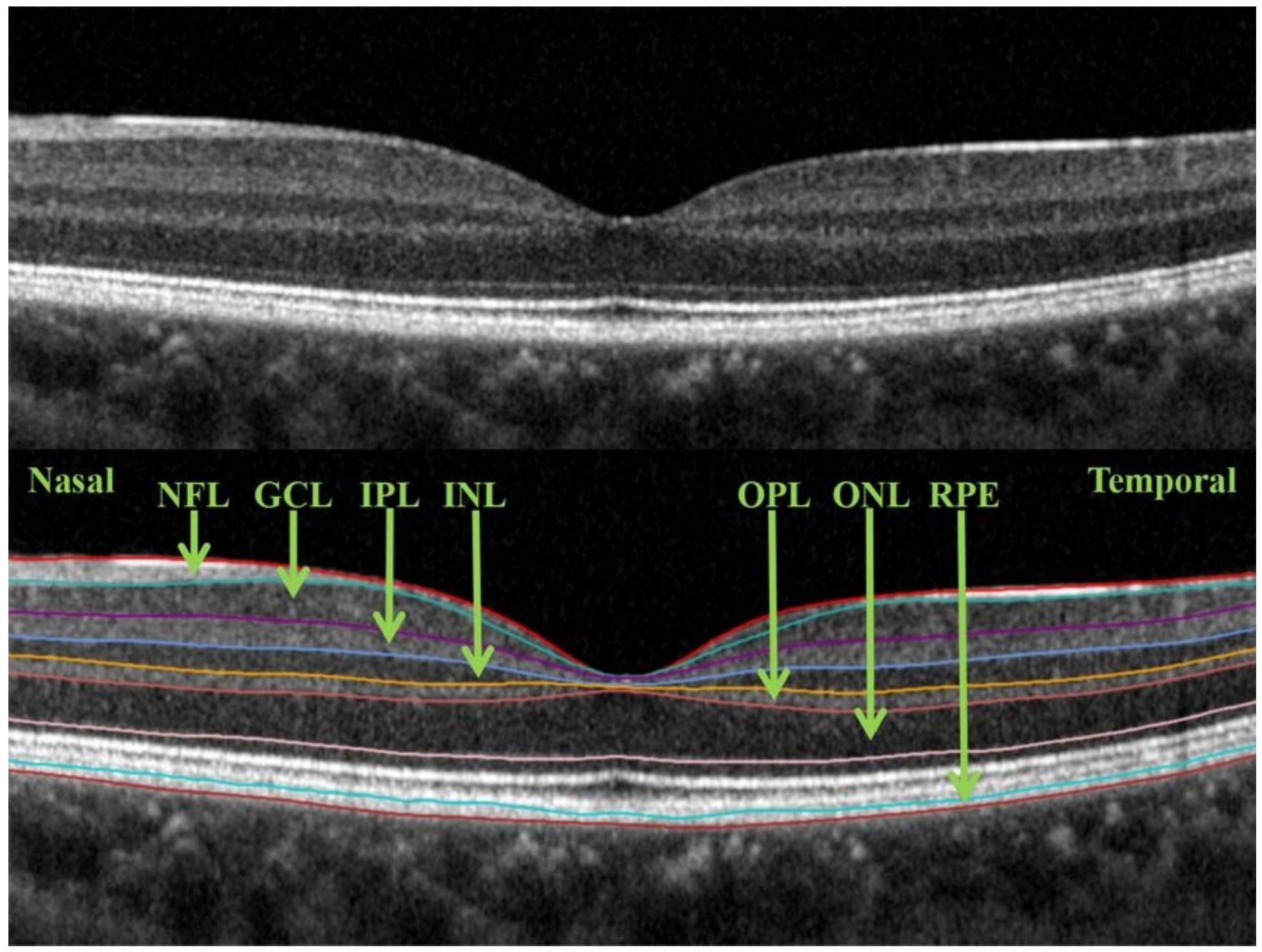

Figure 2

A cross-sectional image acquired using Heidelberg Spectralis spectral-domain optical coherence tomography (upper panel). The automatic segmentation algorithm divides the macula into 7 layers (lower panel). The 7 intraretinal layers include the RNFL = retinal nerve fiber layer; GCL = ganglion cell layer; IPL = inner plexiform layer; INL = inner nuclear layer; $\mathrm{OPL}=$ outer plexiform layer; $\mathrm{ONL}$ = outer nuclear layer; and $\mathrm{RPE}=$ retinal pigment epithelium . 

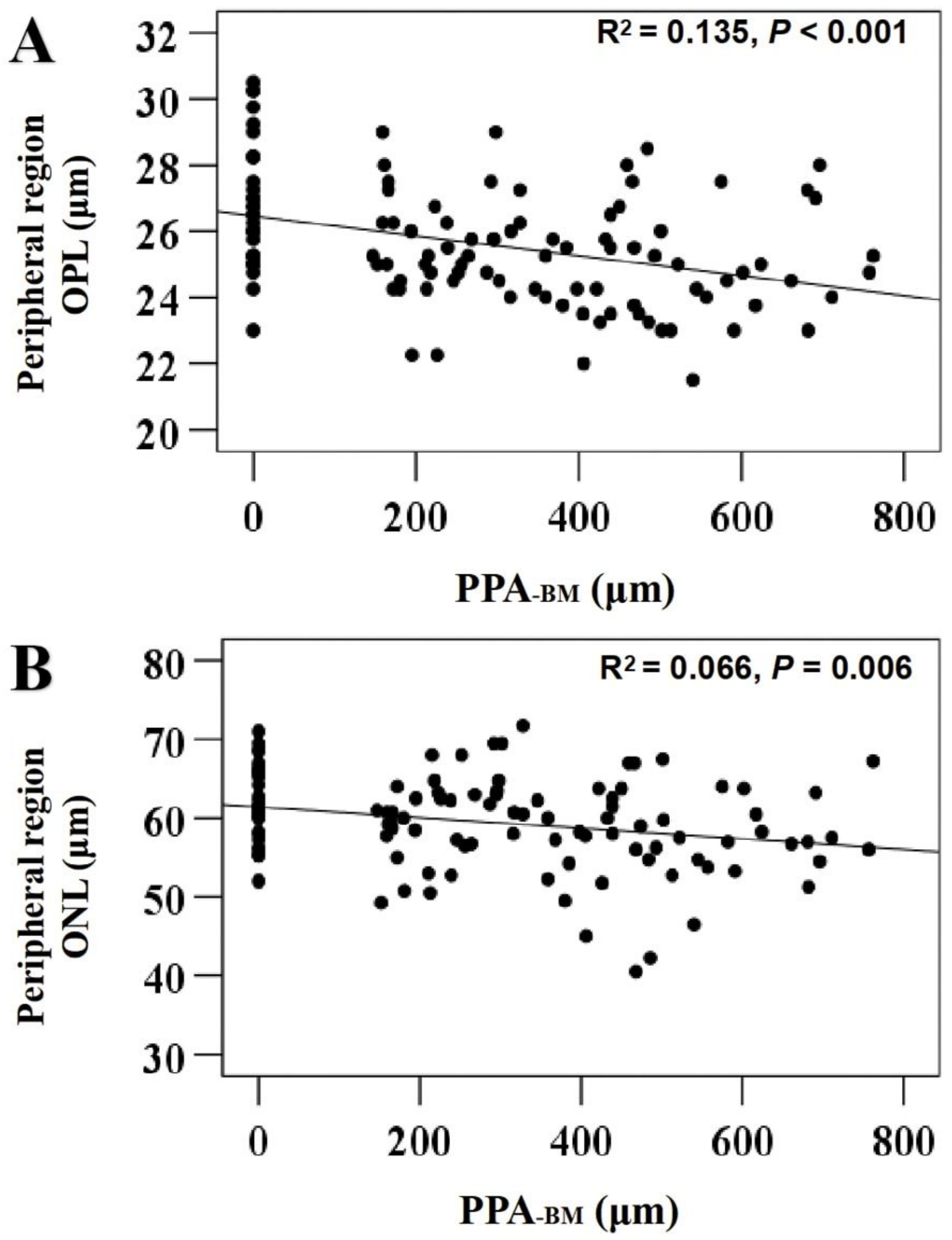

Figure 3

Scatter plots showing the relationship between macular intraretinal layer thickness and $\beta$-parapapillary atrophy with Bruch's membrane (PPA-BM) width. (A) Association between outer plexiform layer (OPL) thickness in the peripheral region and PPA-BM width. (B) Association between outer nuclear layer (ONL) thickness in the peripheral region and PPA-BM width. 